\title{
DEBLURRING STUDY OF DMSP/OLS NIGHTTIME LIGHT DATA BY RTSVD
}

\author{
CHAO REN ${ }^{1,2}, Z H I W E N ~ Y U^{1}, K_{A I Y U A N ~ D E N G}{ }^{1}$, YALONG PAN $^{1}$ \\ 1 College of Geomatics and Geoinformation, Guilin University of Technology, Guilin China - (renchao@glut.edu.cn,yzw8111@163.com, \\ 2761419719@qq.com,shixianjian@glut.edu.cn) \\ 2 Guangxi Key Laboratory of Spatial Information and Geomatics, Guilin China - renchao@glut.edu.cn
}

KEY WORDS: Nighttime light, Deblurring, Regularized, TSVD, L-curve

\begin{abstract}
:
DMSP/OLS, as the earliest Nighttime light remote sensing data, has great application value and can greatly improve the data quality by solving the blurring problem existing in the data. The blur reason is analyzed, and a new algorithm of regularization truncated singular value decomposition (RTSVD) combining with Pct image luminescence frequency filtering is proposed, which can effectively eliminate the blurring phenomenon and retain the real information of the image. Firstly, considering that the luminescence frequency of the light source pixel must be higher than that of the non-light source pixel, the luminescence frequency of the pixel in the Pct image is used to exclude the non-light source pixel in the average light image, and then the truncation parameter of the regularized truncation singular value decomposition (RTSVD) is obtained by using the L curve, so as to decompose and recombine the image. The experiments show that the regularized truncation singular value decomposition method combined with Pct image luminescence frequency filtering can remove the blurring phenomenon on the basis of preserving the image information.
\end{abstract}

\section{INTRODUCTION}

DMSP/OLS(The Defense Meteorological Satellite Program Operational Linescan System) image is a kind of noctilucent remote sensing data widely used, and has important value in social and economic data mining. The image is easy to acquire and can be monitored repeatedly, but improper data collection and management mechanism make the image blurred. Generally, blur is suppressed by selecting threshold or combining other data. Imhoff et al. proposed $89 \%$ cumulative luminescence threshold filtering, which effectively alleviated the problem of light overflow, but only for urban area extraction, and lost a lot of details (Imhoff,1996).Henderson and Sutton et al. obtained the city boundary by setting three different thresholds for DMSP/OLS image, and obtained a relatively accurate city boundary, but the process was too cumbersome(Gu, Gui, 2010. Henderson, 2003). Letu et al. used DMSP/OLS radiation calibration night light intensity data to correct, effectively expanding the dynamic range of intensity values, but not improving the fuzzy phenomenon (Letu,2012).Hao et al. combined NDVI with surface temperature to reduce the saturation of $\mathrm{DN}$ value in the urban center and alleviate the fuzzy phenomenon, but the auxiliary data need to go through complex calculation $(\mathrm{Hao}, 2015)$.Alexei et al. proposed Pct image filtering method (Alex,Oram,2018). Pct image is easy to obtain and the deblurring processing method combined with Pct image filtering is easy to realize. However, Pct image filtering alone is not enough to eliminate the blurring and noise of the light edge, and other methods should be combined.

Firstly, according to the rule that the luminous frequency of the light source pixels must be higher than that of the non-luminous pixels, Pct data was used to compare the luminous frequency of the pixels, close all the non-luminous pixels in the image, and set the threshold of $15 \%$ luminous frequency to eliminate the accidental influence. Then, the gaussian distribution is used to fit the point diffusion function (PSF), and the reflexive boundary conditions are used to construct the fuzzy matrix (Small,2005). Finally, L curve was drawn to calculate the truncation parameters, and the 
regularized truncation singular value decomposition method was used to deblur the image

\section{DMSP/OLS DATA SET}

The DMSP satellite system consists of six satellites and USES a dual-star operation mechanism, with two satellites operating at the same time. The satellite operates in a sun-synchronous orbit with an altitude of $833 \mathrm{~km}$, with an operating cycle of $101 \mathrm{~min}$ and a scanning width of $3000 \mathrm{~km}$. It collects information of 14 orbits every day, which can provide four times of global coverage. OLS sensor is the main sensor of DMSP satellite. OLS sensor has two channels of visible near-infrared (VNIR: $0.4 \sim 1.1$ microns) and thermal infrared (TIR:10 13 microns) (Lieske,1981), with perigee resolution up to $500 \mathrm{~m}$ and scan band edge resolution over $1 \mathrm{~km}$

DMSP/OLS non-radiative calibration night light intensity data include: cloud-free observation frequency image, average light image, stable light image and Pct image. Among them, average light image is the average image of visible light intensity without filtering processing, which is the main research object of fuzzy problem. Stable light image is an image that has been filtered to remove accidental noise. The quality of the image is better than the average light image. The Pct image is the product of the cloud-free observation intensity and the percentage of light detection, and can be used to monitor the light emission frequency.

\section{BLUR REASON OF DMSP/OLS}

\subsection{Data Collection}

The size of fine pixels at the center of mass of the field of view is $0.56 \mathrm{~km}^{*} 0.56 \mathrm{~km}$, much smaller than the field of view area. In the process of field of view movement, the same fixed light source will fall into several overlapping fields of view, forming a fuzzy ellipse with the field of view as the center of the light source. Moreover, due to satellite miscalculation, the center of mass of the fuzzy ellipse has a random deviation, resulting in the geographical position deviation of the light source in the DMSP image. Tuttle et al. calculated the distance and azimuth between the position of the light source and the GPS observation position in the DMSP image every night by moving portable light sources in different parts of the world. All the 28 points measured were migrated northward with an average deviation of $2.9 \mathrm{~km}$, which was about the width of a smooth pixel (Lieske, 1981). (Alexei, 2018) further speculated that the deviation standard deviation was about $1 \mathrm{~km}$ to $1.12 \mathrm{~km}$.

\subsection{Data Management}

At the same time, DMSP satellite cannot directly store high-resolution information of fine pixels, so fine pixels are smoothed when storage, and fine pixels of $5 * 5$ are regarded as a smooth pixel. However, the initial calculation position of DMSP satellite for fine pixel smoothing is not uniform, resulting in no complete overlap of smooth pixel images every night, which is also one of the reasons for the blurring of annual average image.

\section{PCT FILTER AND RTSVD}

\subsection{Pct Filter}

According to the imaging principle of light image, the luminous frequency of the light source pixel must be higher than that of the non-light source pixel. The Pct image can be used to close the pixel whose luminous frequency is less than that of the surrounding pixels to eliminate the pixel error of the light source:

Firstly, check the pixels around $x_{i, j}$, finding the pixel with the highest luminous frequency, $x_{\max }$, and compare $x_{i, j}$ with $x_{\max }$, as follows: 


$$
\left\{\begin{array}{l}
x_{i, j} \geq x_{\max }, x_{i, j}=1 \\
x_{i . j}<x_{\max }, x_{i, j}=0
\end{array}\right.
$$

However, Pct image filtering cannot completely turn off the blurred pixels in the light edge and cannot remove the noise in the image. Therefore, on the basis of Pct image filtering, a regularized truncated singular value decomposition (RTSVD) method was proposed to further process the average light image.

\subsection{RTSVD Algorithm}

Regularized truncated singular value decomposition is a regularized truncated singular value decomposition method, which aims at removing noise and restoring image quality. For row and column fuzzy images, there is generally a linear fuzzy model:

$$
A x=b
$$

Where $x, b$ are the column vectors arranged by the real image and the blurred image respectively, and the length is $N=m n, A$ is a fuzzy matrix, $A \in R^{N \times N}$, The basic deblurring model can be obtained as follows:

$$
x=A^{-1} b
$$

Fuzzy matrix is determined by point spread function and boundary condition. Where, the point spread function can be fitted by using gaussian distribution. According to the blur reason, the boundary conditions of fuzzy matrix are set as reflexive boundary conditions . Then, the singular value decomposition is used to further improve the de-fuzzy model. For $m \times n$ order fuzzy matrix, there is a decomposition such that:

$$
A=U \Sigma V^{T}
$$

Where $U$ and $V$ are orthogonal matrices, respectively are left singular vector matrix and right singular vector matrix, which satisfy $U^{T} U=I_{m}, V^{T} V=I_{n} . \Sigma_{\text {is }}$ the singular value matrix, $\sigma_{1}, \sigma_{2} \ldots \sigma_{m}$ is the diagonal matrix, is the singular value on the diagonal, satisfies $\sigma_{1} \geq \sigma_{2} \geq \cdots \geq \sigma_{m} \geq 0$. Formula (3) can be written as: $x=A^{-1} b=V \Sigma^{-1} U^{T} b=\Sigma_{i=1}^{m} \frac{u_{i}^{T} b}{\sigma_{i}} v_{i}$
Where, $u_{i}$ is the left singular vector and $v_{i}$ is the right singular vector. The fuzzy matrix $A$ is ill-conditioned matrix with a very large number of conditions, $\operatorname{cond}(A)=\sigma_{1} / \sigma_{n}$ and a very fast decay rate of the singular value. The error component corresponding to the small singular value is amplified to interfere with the real information of the image. The formula is described as follows:

$$
x=\sum_{i=i}^{m}\left(\frac{u_{i}^{T} b_{0}}{\sigma_{i}}+\frac{u_{i}^{T} \Delta}{\sigma_{i}}\right) v_{i}
$$

Where, $\Delta$ is error. In order to weaken the error interference, the regularized truncated singular value decomposition method is used to replace the original singular value matrix $\sum_{m}$ with the truncated singular value matrix $\sum_{k}$, and the formula is as follows:

$$
x_{k}=\sum_{i=1}^{k} \frac{u_{i}^{T} b}{\sigma_{i}} v_{i}, k<m
$$

Where, $k$ is the truncation parameter. The error of the regularized truncated singular value decomposition method comes from regularization and noise.

\subsection{L-curve}

The selection of truncation parameters is very important for RTSVD algorithm, and GCV or L curve is generally used to determine the truncation parameters. However, it is difficult for GCV to calculate the minimum truncation error when the image is blurred. Therefore, $\mathrm{L}$ curve is used to determine the truncation parameters and cubic spline curve is used to fit the discrete points. L curve is a curve drawn for a series of truncation parameters in the rectangular coordinate system with canonical solution norm as the vertical axis and the corresponding residual norm as the horizontal axis on the scale of log-log. The truncation parameter corresponding to the point of maximum curvature on $\mathrm{L}$ curve is the optimal solution. Let $\rho, \eta$ are the logarithm of the residual and solution respectively, and the curvature formula of $\mathrm{L}$ curve is expressed as follows:

$$
K=\frac{\rho^{\prime} \eta^{\prime \prime}-\rho^{\prime \prime} \eta^{\prime}}{\left[\left(\rho^{\prime}\right)^{2}+\left(\eta^{\prime}\right)^{2}\right]^{3 / 2}}
$$

In truncated singular value decomposition, $\mathrm{L}$ curve is composed of several discrete points. To calculate the optimal solution, a differentiable and smooth curve associated with discrete points must be defined under 
the condition that the overall shape of the curve remains unchanged. Therefore, cubic spline curve was used to approximate the discrete point of L curve, and the maximum curvature point on the continuous curve was calculated to determine the truncation parameter.

\section{EXPERIMENTAL RESULTS AND ANALYSI}

In order to verify the effectiveness of regularized truncated singular value decomposition (TSVD) in
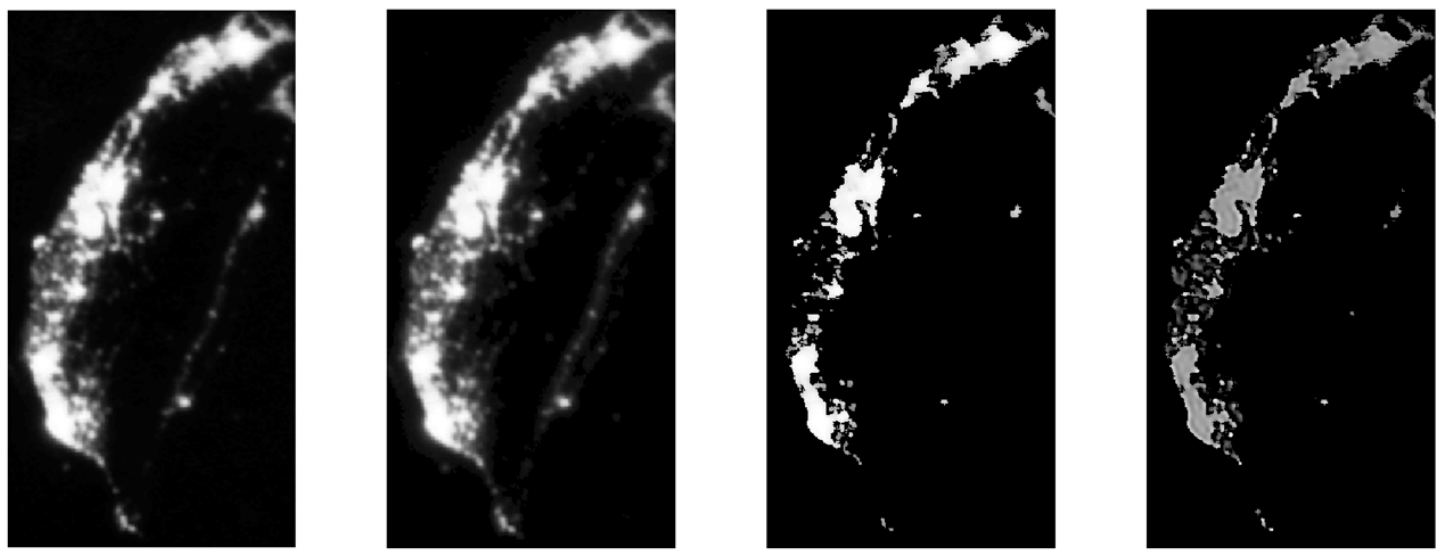

Figure 1. DMSP/OLS average light image deblurring

Table 1. Deblurring evaluation

\begin{tabular}{ccccc}
\hline Images & PSNR & Image entropy & GMG & Edge strength \\
\hline Original image & 23.1727 & 0.8938 & 0.7582 & 8.4183 \\
Stable lights & 24.7278 & 0.9753 & 0.7820 & 8.2769 \\
Cumulative threshold image & 26.6162 & 0.4110 & 2.8530 & 30.5899 \\
Proposed method & 25.6780 & 1.0912 & 1.7821 & 18.2736 \\
\hline
\end{tabular}

In addition to visual observation and evaluation of experimental results, peak signal-to-noise ratio (PSNR), image information entropy, grayscale mean gradient (GMG) and edge intensity were introduced as objective evaluation criteria. It can be seen from table 1 that the original average light image (a) has a serious ambiguity. The truncation parameter was selected according to the $\mathrm{L}$ curve. Compared with the original image, the processed image (d) showed that the peak signal-to-noise ratio increased by 2.5053 , the information entropy increased by 0.1974 , the average grayscale gradient increased by 1.0239 , and the edge intensity increased by 9.8553.For the image (c) processed by direct frequency filtering, the peak signalto-noise ratio was increased by 3.4435 and the information entropy was reduced by 0.4828 , indicating deblurring DMSP images, the average light image of F142000 in Taiwan area with a size of $417 * 223$ pixels was cut out for deblurring. In order to evaluate the effect of the method in this paper, stable light image and $89 \%$ cumulative luminescence threshold filter image were selected for comparison with the experimental results, as shown in figure 1. 
calculated by fitting the discrete points of $\mathrm{L}$ curve with the spline curve.

(3) RTSVD algorithm can keep the details of the image while removing the blur.

\section{REFERENCES}

Abrahams, A., Oram, C., Lozano-Gracia, N., 2018. deblurring dmsp nighttime lights: a new method using gaussian filters and frequencies of illumination. Remote sensing of environment, 210, 242-258.

Gu Y., Gui Q., 2010. A new method for choosing truncated parameters in tsvd solution, Journal of Surveying and Mapping Science and Technology, 27(3), 176-179.

Henderson M., Yeh E., Gong P., Elvidge, C., Baugh, K., 2003. Validation of urban boundaries derived from global night-time satellite imagery. Remote Sensing, 24(3): 595-609.

Hao, R., Yu, D., Sun, Y., Cao, Q., Liu, Y., Liu, Y., 2015. Integrating multiple source data to enhance variation and weaken the blooming effect of dmsp-ols light. Remote Sensing, 7:1422-1440.

Hansen, P., Nagy, J., O'leary, D., 2006. Deblurring images: matrices, spectra, and filtering (Vol. 3). Siam.

Hansen, P., O'Leary, D., 1993. The use of the L-curve in the regularization of discrete ill-posed problems. SIAM Journal on Scientific Computing, 14(6), 14871503.

Imhoff M., Lawrence W., Stutzer D., Stutzer, D., 1999. A technique for using composite dmsp/ols "city light" satellite data to map urban area. Remote Sensing of Environment, 61(3): 361-370.

Letu H, Masanao H, Tana G, Nishio, F., 2012. A saturated light correction method for dmsp/ols nighttime satellite imagery. IEEE Transactions on Geoscience and Remote Sensing, 50(2):389-396.
Lieske, R., 1981. DMSP primary sensor data acquisition. International Foundation for Telemetering.

Lin D., Zhu J., Song Y., 2017. Methods for determining tsvd truncation parameters considering the influence of truncation deviation. Acta Geochimica Sinica, 46(6), 679-688.

Li C., Zheng Y., Zhang C., Han J., Wang L., 2013. Comparative analysis of regularization methods for fish-eye camera calibration. Bulletin of Surveying and Mapping, 2013(12), 36-40.

Liu J., Jiang T., Jiang G., Jia B., 2012. Solution of rational polynomial parameters based on l-curve method and gcv method. Bulletin of Surveying and Mapping, 2012(S1), 330-333.

Sutton P, Roberts D, Elvidge C, Meij, H., 1997. A comparison of nighttime satellite imagery and population density for the continental United States. Photogrammetric Engineering and Remote Sensing, 63(11):1303-1313.

Small, C., Pozzi, F., Elvidge, C., 2005. Spatial analysis of global urban extent from DMSP-OLS night lights. Remote Sensing of Environment, 96(3-4), 277-291.

Tuttle, B., Anderson, S., Sutton, P., Elvidge, C., Baugh, K., 2013. It used to be dark here. Photogrammetric Engineering \& Remote Sensing, 79(3), 287-297. 